\title{
Testbeds for Assessing Critical Scenarios in Power Control Systems
}

\author{
Giovanna Dondossola ${ }^{1}$, Geert Deconinck ${ }^{2}$, Fabrizio Garrone ${ }^{1}$, \\ and Hakem Beitollahi ${ }^{2}$ \\ 1 CESI RICERCA, Milano, Italy \\ 2 K.U. Leuven ESAT, Kasteelpark Arenberg 10, 3001 Leuven, Belgium \\ dondossola@cesiricerca.it
}

\begin{abstract}
The paper presents a set of control system scenarios implemented in two testbeds developed in the context of the European Project CRUTIAL - CRitical UTility InfrastructurAL Resilience. The selected scenarios refer to power control systems encompassing information and communication security of SCADA systems for grid teleoperation, impact of attacks on inter-operator communications in power emergency conditions, impact of intentional faults on the secondary and tertiary control in power grids with distributed generators. Two testbeds have been developed for assessing the effect of the attacks and prototyping resilient architectures.
\end{abstract}

Keywords: power control systems, SCADA systems, grid teleoperation, voltage and frequency control, inter-utility communications, power emergency conditions, cyber security, resilient architectures.

\section{Introduction}

In the context of the protection of critical information infrastructures, the need of addressing infrastructures owned, operated and used by the power utilities is considered fundamental to the security, economy and quality of life at national and international level [1. Electricity market liberalisation, energy revolution and technology breakthroughs are three determining factors in the introduction of advanced networked systems for the security and adequacy of modern Electric Power Systems. However networks based on Information and Communication (ICT) technologies create a lot of interdependencies among geographically distributed infrastructures controlled by multiple stakeholders, which motivates research and technology developments able to reduce the cyber risk and to defend power utility infrastructures from intentional and accidental threats.

A wide set of control system scenarios has been identified by the CRUTIAL project [2], presenting how dependencies among (segments of) power, control and information infrastructures enable propagation of failures and appearance of cross-cascading and escalading phenomena [3]. Two CRUTIAL testbeds are under development in the CESI RICERCA and K.U.Leuven research laboratories at the aim to assess the ICT system's vulnerability to plausible cyber attacks and evaluate the resilience of possible architectures/mechanisms/solutions 
to such threats [4. A subset of CRUTIAL scenarios within both testbeds has been selected for presentation in the present paper, according to the following structure. Section 2 focuses on scenarios related to the DSO (Distribution System Operator) and TSO (Transmission System Operator) teleoperation systems, while section 3 presents scenarios related to control systems for distributed energy resources. Sections 4 and 5 describe both testbeds.

\section{Security in Hierarchical Power Control Systems}

Two CRUTIAL interdependency scenarios from [2] are presented covering several control systems involved in both manual teleoperations and automatic emergency management of the power grid.

\subsection{Communication Security of Grid Teleoperation}

The main purpose of this scenario consists in the assessment of the security of the ICT components involved in the teleoperation activities of a DSO operator through the analysis of cross-cascading effects due to threat occurrence in both normal and abnormal power conditions. In general terms a SCADA system for the grid teleoperation is working when it is able to perform its monitoring and control functions. This may happen when the power service is stable (for operational and maintenance needs), but also in abnormal or even emergency conditions. The teleoperation activity includes:

- continuous monitoring of substation status: information flows from substation to centre, part on a continuous base (measurements related to active power $\mathrm{P}$, reactive power $\mathrm{Q}$, voltage $\mathrm{V}$ and breaker positions) and others (e.g. alarms and status variations) as asynchronous flows.

- interventions on the grid configuration (opening/closing breakers, line trips, etc.) due to several needs such as predictive maintenance, DSO contingency management and preventive control requested by the TSO (like rotating load shedding plans and variations in transformer tap changers).

The core ICT-systems involved in the supervision and control activities are: the ATC-Area Telecontrol Centres (whose operator's console is shown in figure 1) controlling the power substations; the substation automation systems connected to their centres through Wide Area Networks (WAN).

By following an ongoing trend in the utilities' communication technology renewal, the information flow supporting DSO teleoperation is transmitted by standard telecom IP backbones owned and operated by external providers who supply virtual, dedicated channels over communication links shared with other customers. From the DSO perspective such a communication infrastructure may be targeted by security threats sourced within the Telco infrastructure. Due to the strong availability requirements on the communication system (availability equal to 0.99999), redundant communication paths are used, implemented over physically independent carrier lines, possibly owned by distinct telecommunication providers. The correct execution of the DSO teleoperation requires the 


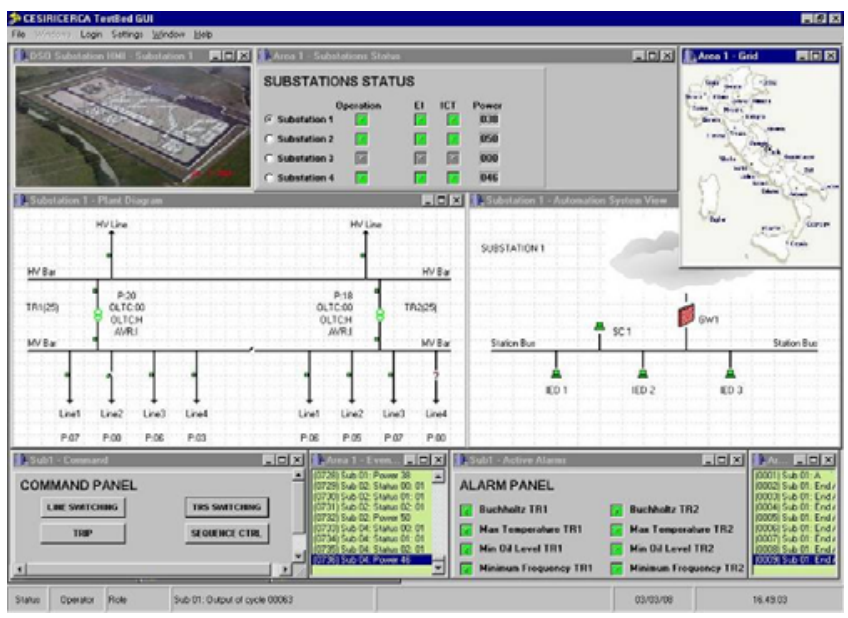

Fig. 1. DSO Operator's Console

satisfaction of strict refresh time requirements for measurements/alarms and delivery time requirements for commands to the operator.

ICT threats that may affect the communication infrastructure range from Denial of Service (DoS) attacks to the telecontrol communications and intrusions into the centre/substation communication flow eventually followed by the execution of faked commands troughs the exploitation of the vulnerabilities of the standard application layer protocols used for monitoring activities and command transmission [2]. DoS attacks to the teleoperation communications, generated by enemies located on the telecom IP backbone are being explored first. Such DoS attack processes to IPv4/IPSEC channels target both DSO centres and substation routers/gateways/firewalls. The identified attack plan includes a sequence of attacks showing the increasing severity of DoS processes starting from the denial of the supervision function and maintenance activities, toward precluding the DSO operator from properly doing the contingency management, towards the denial of the execution of the defence actions in pre-emergency conditions.

The simulation of attack processes on the testbed will allow i) to assess the capability of the secure and redundant communication architecture to tolerate the threat hypotheses and evaluate the possible cascading effects in presence of power contingencies; ii) to assess the vulnerabilities of the activities based on standard protocols (e.g. IEC 60870-5-104); iii) to assess the sharing of the same channel for real-time and not real-time activities.

The severity of the cascading effects proved in the laboratory testbed is expected to vary depending on i) the grid operating conditions during the ICT attacks ii) the number of substations involved iii) the level of urgency of the teleoperation intervention. In normal conditions, an attack to a single substation site is not expected to lead the power system in a critical status. However if 
the DSO operator is repairing a previous contingency the consequence of the attack may provoke a delay in the duration of the power service interruption. Even worse if the operator's intervention is aimed at facing with pre-emergency situation occurred in the higher level grid, the impossibility to perform the defence action may lead the whole system into troubles.

\subsection{Impact of Attacks on TSO Emergency Management}

The realisation of technologically integrated defence plans requires that in emergency conditions the TSO is authorised by the DSO to activate defence actions, consisting in the automatic execution of load shedding activities on the distribution grid. This scenario explores the security of the communications between the TSO and DSO under emergency operating conditions (i.e. overloading of power lines), assessing the possible cross-cascading effects of ICT threats to the communication channels connecting TSO and DSO control centres and substations.

The TSO control centre monitors the Electric Power System and elaborates some potentially emergency conditions that could be remedied with opportune load shedding actions applied to particular areas of the grid. In order to actuate the defence actions the TSO centre chooses a subset of $\mathrm{HV} / \mathrm{MV}$ substations from the list of substations participating to the emergency plan, then sends the requests of preventively arming the automation devices of these substations to the interested DSO area control centres. These requests are delivered through a communication channel among a TSO centre and the interested DSO centres. The DSO centres provide for arming the required substations, and return their status to the TSO centre. In case the potential emergency condition evolves into a real emergency situation, a TSO sentinel device sends the trip command which has to be delivered through the communication network within $600 \mathrm{~ms}$ to recover the emergency. It is worth of notice that TSO arm requests are asynchronous with respect to the trip commands. The objective of the TSO is to maintain the electric power system in a secure state. In order to prevent the escalation of a possible emergency situation, the TSO Energy Management System makes frequently a selection of detachable loads and emits arm requests to their corresponding control centres.

The investigated threats are the same of the previous scenario, but the Communication Network is more complex because it interconnects two separate (TSO and DSO) teleoperation networks. Cyber attacks carried out under emergency conditions, when defence actions have to be performed out under strict real time constraints, can cause severe damages, e.g. inhibiting the proper execution of the required automatic load shedding actions may provoke the degeneration of the emergency in the transmission grid. The effects on the whole power system of the considered ICT attacks will depend on the number of components involved. As in the previous case the severity of ICT-Power cascading effects depends on the specific sequencing of attacks during the ongoing emergency procedure. 


\section{Control Vulnerabilities for Distributed Energy Resources}

The penetration of distributed generation in the electricity grid is increasing [5]. For optimal deployment of distributed energy resources (DER, such as generators and storage units), the underlying control applications are also distributed and require communication among the intelligent electrical devices (IED) 6. Therefore, it is necessary to investigate the impact of different types of ICT anomalies on this communication network and on the control applications and hence to identify the vulnerabilities. Examples of such anomalies include physical (random) faults and intrusions (malicious faults). To this extent, a 16-node radial segment of a grid with several DER has been simulated together with its control algorithms (figure 2). The simulation has been set-up as set of communicating Java processes, running in a Linux environment on a workstation PC. Via configuration file, the electrical topology and parameters can be set. In each timestep, the resulting electrical equilibrium is calculated, as generator and load profiles are changing.

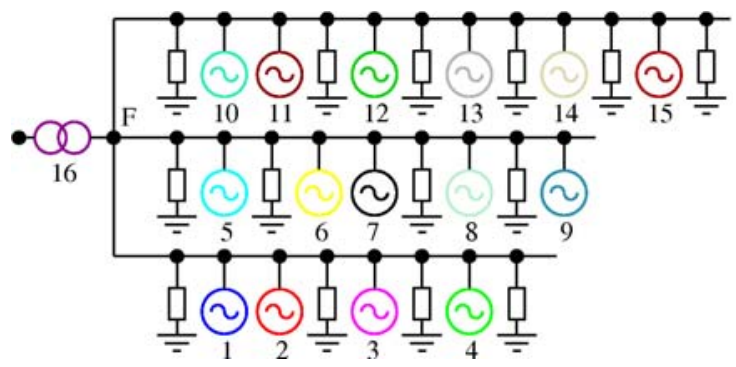

Fig. 2. Radial distribution segment with DER used in simulations

This simulated distribution grid segment has 3 branches with 15 nodes (each representing a generator and a load) and is connected via a transformer to the higher level electricity grid. Three control applications are integrated. A primary control algorithm controls active power output based on local voltage level only, i.e. it does not require communication. Frequency is kept stable by the connection to the external power grid via node 16. Secondary control (keeping the voltage and frequency within its limit) and tertiary control (optimising economically), however, are based on communication among the nodes. In the simulation, a decentralised approach has been chosen for this communication, in which IED of loads and generators of the radial distribution segment use an overlay network that is set up on top of the physical communication infrastructure 788 . Such overlay network allows to deal with random faults as well as with dynamic changes in the topology $[9$. Secondary and tertiary control is based on a gossiping algorithm on top of the overlay network. It is assumed that communication 
delays and gossiping intervals are at least an order of magnitude larger than the time needed for settlement of the primary control loop. In the simulations, this means that power flow calculations and the primary control actions are calculated first until convergence is observed. Only then, IED associated to generators will gossip, and adjust their parameters for the primary control loop according to the results of secondary and tertiary control loop. When all generators finished gossiping, new power flow calculations are done until convergence, and so on. The number of iterations is chosen in advance.

For tertiary control purposes, cost curves are associated to generators, which are chosen to be monotonically increasing linear functions with a marginal cost for zero output (which is not necessarily zero) and some marginal cost for the generator maximum output. The feed-in transformer has no bounds on the amount of power it can inject into the distribution net (which is realistic for the power levels in the presented DER scenarios). The transformer has a relatively high marginal cost curve which increases when injected power increases. This high price favours local generators to produce.

In all scenarios presented a similar load profile is applied. Generators start from set point $P_{0}$ (desired power output) and loads have a fixed consumption. Unbalances between local supply and demand are automatically dealt with by the transformer. At time instance (or iteration) 21 several loads increase consumption and at time instance 121 several loads decrease it. Hence, one will typically observe three phases during a simulation:

$-t=[1 . .20]$ : Steady settlement of initial settings to global optimum.

$-t=[21 . .120]$ : Demand increases; initially, feeder resolves unbalance and some distributed generators react as well (if local voltage drop is high enough). Afterwards, they adjust power output to evolve towards optimum.

- $t=$ [121..181]: Demand suddenly decreases; again, feeder and some distributed generators resolves the unbalance, after which all power outputs are adjusted towards new optimum.

Simulation results are displayed using three graphs, showing information on all generators and the feeder transformer at every time step: active power output $P$, voltage levels $V$ and marginal costs $C$ of each generator.

The simulations have been performed, first without ICT anomalies (reference results not shown here), and consequently, in different experiments, subject to several threats on the communication network among the IED.

\subsection{Denial-of-Service Attacks on IP-Network}

A denial-of-service (DoS) attack tries to disturb the functionality of service by flooding a service provider with fake or unfinished requests. Such DoS can be generic (caused by a worm attacking random computers/networks) or targeted (e.g. by constantly joining and leaving the overlay network, which triggers a bandwidth consuming algorithm searching new neighbours) and may lead to a denial of all communication over one or more channels. Whatever underlying reason or mechanism for the DoS attack, it results in long communication delays, which lead 

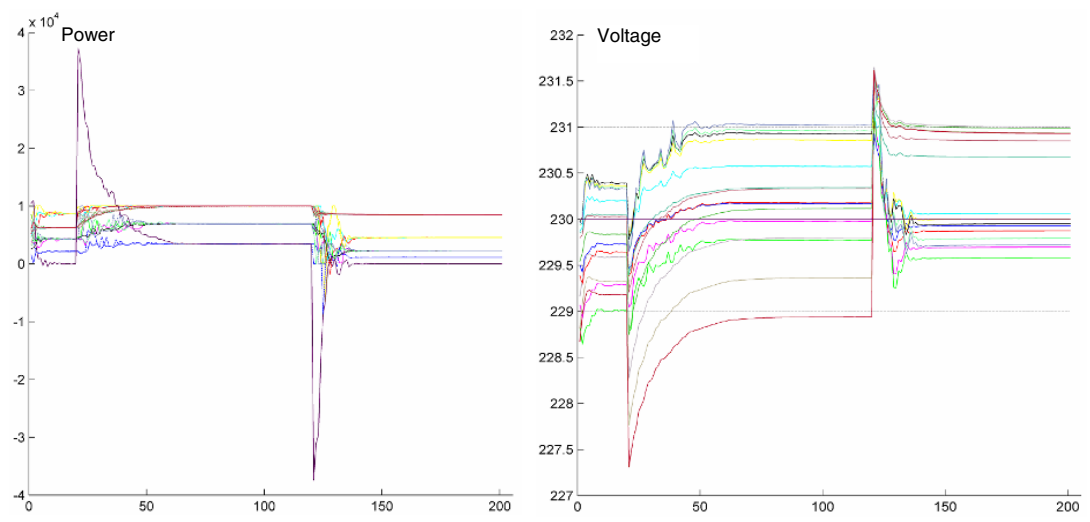

Fig. 3. Power output and voltage level when overlay network is partitioned

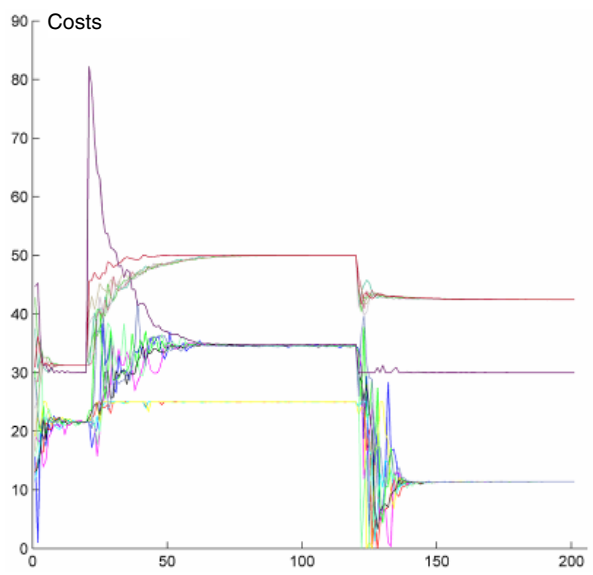

Fig. 4. Marginal production costs when overlay network is partitioned

to loss of connection between IED, while they are participating in secondary and tertiary control schemes. As such, the system degenerates to distribution segment in which some generators are not taking part in control applications. Hence secondary and tertiary control algorithms will not converge to a global optimum, but rather seek an optimal solution among participating IEDs.

\subsection{Attack on Overlay Network Topology}

A different scenario is an attack on the topology of the overlay network by some malicious node(s). To set up and maintain the overlay network, such malicious nodes would send fake results to nodes searching for new neighbours as to make themselves the new neighbour of these nodes. After some time, these malicious 
nodes become a centre of the overlay network. The overlay network may partition into separate parts as a result of such malicious attack to the overlay network (or also as a result of a major communication infrastructure, which partitions the underlying physical network). In the simulation, the overlay network partitions in two groups: i) IED of generator 1 to generator 9, and ii) IED of generator 10 to generator 15 with the grid-connected transformer. Note that the system remains connected electrically.

The influence of the splitting of the overlay network results in two groups of generators that are locally converging - but not globally (figure 3). For the cost curve (figure 4), this results in convergence to two different cost levels, while a single equal marginal cost for all generators is the global optimum.

\subsection{Voltage Level Attack}

As indicated above, the secondary control algorithm implemented among the IED optimizes voltage levels in all points of the distribution grid segment as to minimize the divergence from rated values. Since over-voltages can damage equipment attached to the power grid, attacks on the secondary control loop can induce over-voltages which can trigger the protection, leading to local black-outs, or physically damage grid assets. A malicious node could inject false values in the secondary control loop (which is based on a distributed averaging algorithm on top of the overlay network). Over time, these errors accumulate and the global average will diverge from its correct value, leading to incorrect IED set points.

The simulation shows the result of a malicious IED incorrectly injecting large values into the distributed averaging algorithm of the secondary control loop. Such large value normally means that voltage levels are low in most parts of the distribution grid, and this encourages the other generators to increase active power production. Figure 5 shows that all local generators increase their production, and that the power output of the feeding transformer decreases below
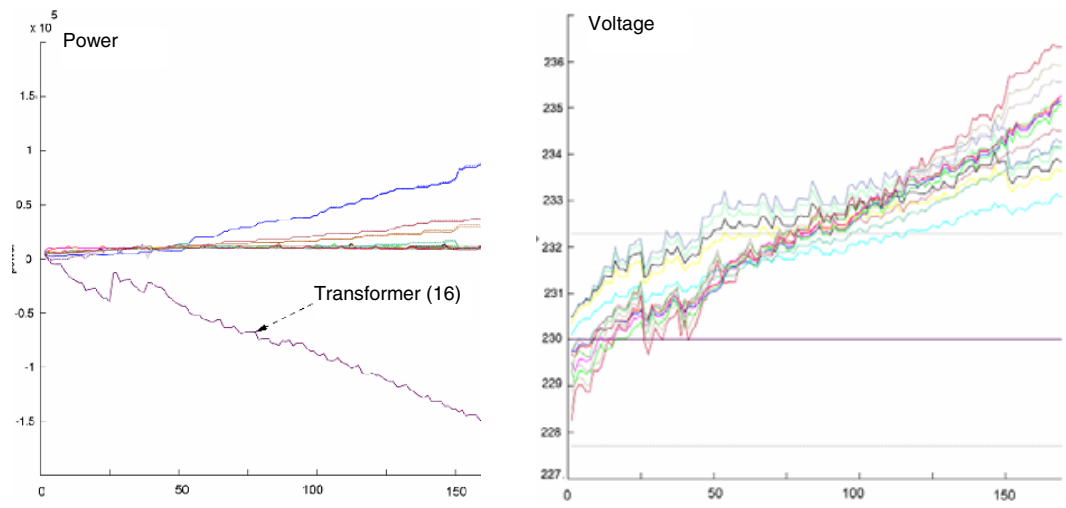

Fig. 5. Power output and voltage levels when malicious node injects incorrect values, resulting in a voltage level attack 
zero, meaning that excess power flows back to the higher level grid. However, these increasing power injections also increase local voltage to dangerous levels, and thus the malicious node succeeded into a voltage level attack.

\section{Resilience Assessment of Teleoperation Systems}

The laboratory testbed for teleoperation systems realises a prototypal but significant power system management architecture with its integrated ICT infrastructure. Focus is being placed on the development of those aspects of the actual power control system which can be used for the implementation of a set of significant attack scenarios, in order to evaluate their plausibility, to demonstrate the possible evolution of the attack processes and to assess the severity of the potential damage on the attack's targets. Besides the two scenarios described in section 2 , the testbed architecture deploys other two interdependency scenarios described in 4 addressing security issues arisen in the integration of operation and maintenance data and in the centralised maintenance of ICT devices, including communication and control devices.

The communication architecture is based on the following assumptions:

- the two lower layers of the OSI stack (physical and datalink) are modelled by switched Ethernet, both for local and wide area communications;

- TCP/IP and UDP/IP are used at the transport/network layers;

- application layer data exchange does not make use of commercial protocols, but the contents of the Application Protocol Data Units (APDU)s are compliant with the appropriate standard (IEC 60870-6 Inter-Control Centre Communications Protocol-ICCP/TASE-2, IEC 60870-5-104 for centresubstation communications, IEC 61850 for communications within the substations).

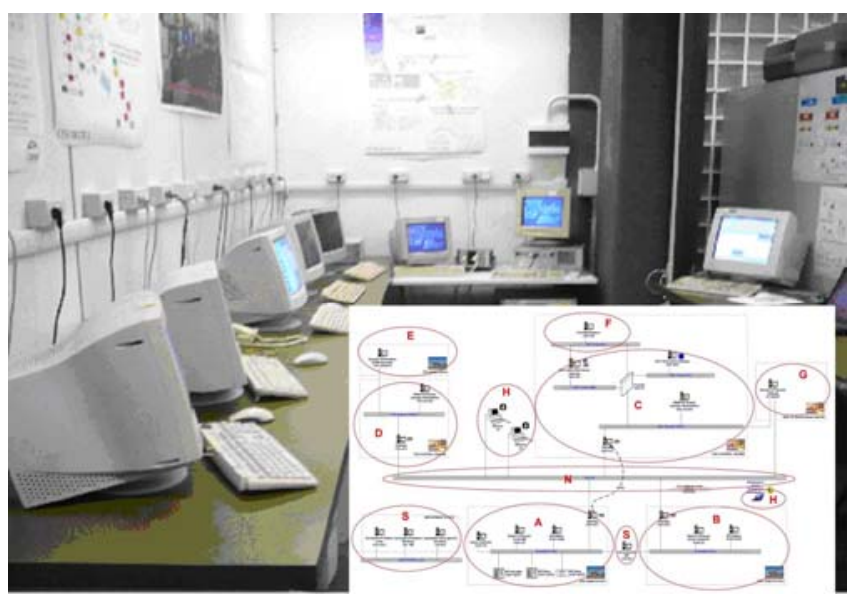

Fig. 6. Grid teleoperation testbed 
Figure 6 gives the layout of the testbed platform implementing the TSO and DSO teleoperation of two high-medium voltage substations.

\section{Testbed for Vulnerability Assessment of DER Control}

A high penetration of DER has a considerable impact on the electrical and control aspects of the grid [1011, as well as it provides many opportunities for distributed control [12 13 14]. In order to test cyber problem scenarios [1516] in DER control applications presented in section 3 - based on simulation - on a real setup, a laboratory testbed has been built, consisting of IED - implementing the control and communication - controlling power electronic converters - which are connected electrically in a microgrid. These converters emulate distributed energy resources, such as a small-scale electricity generator (photovoltaic systems, wind turbines), a load (possibly voltage/frequency dependent), energy storage devices (e.g. a battery, fuel cell). The IED are responsible for the primary control of the converter, but also for secondary and tertiary control algorithms on top of the communication network.

The platform, consisting of converters and IED, allows control applications to be modelled in a high level programming tool (Matlab/Simulink), after which they are downloaded onto the hardware for execution on the created microgrid [17] This Matlab/Simulink interface also provides a real-time interface on the IED to the physical electronic hardware, in order to monitor and control it. The IED are based on industrial Linux-based PC, extended with the realtime framework Xenomai 18. These Matlab/Simulink tools on the different IED associated to different converters are interconnected by standard communication technology (Ethernet and TCP/IP). This set-up allows analysing effects of different types of faults in the communication network on the electrical control applications (secondary and tertiary control, data aggregation, power quality monitoring and mitigation, demand side management, etc.).

As such, this testbed evaluates dependencies of the electric power system from the information infrastructure, and identifies the robustness of the control algorithms to disturbances (figure 7).
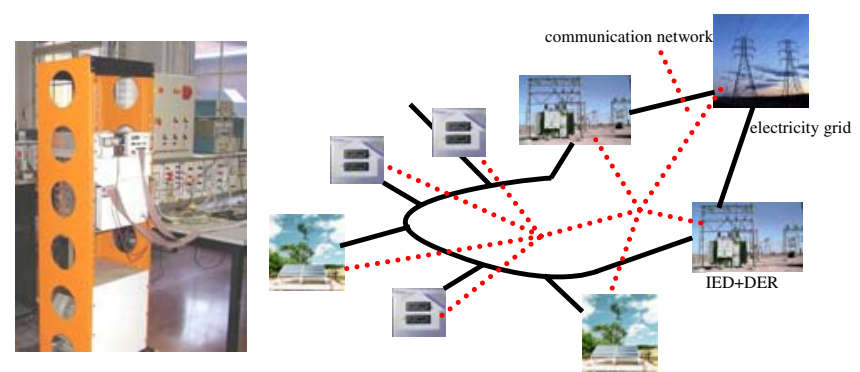

Fig. 7. DER testbed: converter platform (left) and setup of DER interconnected electrically (thick lines) with corresponding IED interconnected via communication (dashed) 


\section{Conclusions}

The paper presents intermediate results of the activities undertaken within the European Project CRUTIAL related to the development of testbeds for assessing the impact of ICT threats on power control systems.

The K.U.Leuven microgrid testbed is set up to evaluate behaviour of ICTdriven decentralised control algorithms in microgrids with a large penetration of DER. By interconnecting IED over ICT infrastructure - beside interconnecting DER electrically, it is possible to evaluate opportunities and vulnerabilities of such coupled infrastructure. In future work, this ICT infrastructure will integrate CRUTIAL middleware modules to make more robust to different types of faults. The testbed will be used to assess and analyse their effect on the microgrid control behaviour.

The CESI RICERCA testbed addresses both concrete needs and envisaged evolutions of power grid control systems. The testbed scenarios evaluate at which extent complex control infrastructures implementing multiple operators' defence actions can be protected by resilient ICT architectures. The scenarios cover emerging themes like information and communication security aspects of power substation control, support to emergency management by the distribution grid control, interactions between process control and corporate activities and remote maintenance of ICT automation devices. The testbed architecture reflects the WAN of LANs communication topology of the CRUTIAL reference architecture, including VPNs and firewall filtering techniques. The Human Machine Interface applications supporting the scenario evolution within the CRUTIAL testbed enrich the typical supervision and control functionality currently available in the control rooms with several ICT-related information that may increase the situation awareness of the operators and their capability to promptly recovery from ICT-enabled power failures. In this respect the testbed activity allows to improve the human aspects involved in the whole power system resilience.

Acknowledgements. This work has been partially financed by the European Commission with the IST Project 27513 CRUTIAL http://crutial.cesiricerca.it.

\section{References}

1. Abele-Wigert, I., Dunn, M.: An Inventory of 20 National and 6 International Critical Information Infrastructure Protection Policies. In: International CIIP Handbook 2006, vol. I. Center for Security Studies, ETH Zurich (2006)

2. Garrone, F., Brasca, C., Cerotti, D., Raiteri, D., Daidone, A., Deconinck, G., Donatelli, S., Dondossola, G., Grandoni, F., Kaâniche, M., Rigole, T.: Analysis of new control applications. CRUTIAL Workpackage 1 Deliverable D2. CRUTIAL consortium (2007)

3. Rinaldi, S., Peerenboom, J., Kelly, T.: Identifying, understanding, and analyzing critical infrastructureinterdependencies. IEEE Control Systems Magazine 21(6), 11-25 (2001)

4. Deconinck, G., Beitollahi, H., Dondossola, G., Garrone, F., Rigole, T.: Testbed deployment of representative control algorithms. Technical report CRUTIAL Workpackage 3 Deliverable D9. CRUTIAL consortium (2008) 
5. Kueck, J., Kirby, B.: The distribution grid of the future. The Electricity Journal (Elsevier Science), 78-87 (June 2003)

6. Deconinck, G.: An evaluation of two-way communication means for advanced metering in Flanders (Belgium). In: Proceedings of the IEEE Int. Conf. on Instrumentation and Measurement Technology (I2MTC 2008), Victoria, Vancouver Island, Canada, pp. 900-905 (2008)

7. Vanthournout, K., De Brabandere, K., Haesen, E., Van Den Keybus, J., Deconinck, G., Belmans, R.: Agora: Distributed tertiary control of distributed resources. In: Proceedings of the 15th Power Systems Computation Conf. (PSCC 2005), Liege, Belgium (2005)

8. Vanthournout, K., Deconinck, G., Belmans, R.: A middleware control layer for distributed generation systems. In: Proceedings of the IEEE Power Systems Conference and Exhibition (PSCE 2004), New York City, USA (2004)

9. Deconinck, G., Rigole, T., Beitollahi, H., Duan, R., Nauwelaers, B., Van Lil, E., Driesen, J., Belmans, R., Dondossola, G.: Robust overlay networks for microgrid control systems. In: Proceedings of the Workshop on Architecting Dependable Systems (WADS 2007), co-located with 37th Ann. IEEE/IFIP Int. Conf. on Dependable Systems and Networks (DSN 2007), Edinburgh, Scotland, UK, pp. 148-153 (2007)

10. Vu Van, T., Driesen, J., Belmans, R.: Power quality and voltage stability of distribution system with distributed energy resources. Int. Journal of Distributed Energy Resources 1, 227-240 (2005)

11. Hadjsaid, N., Canard, J., Dumas, F.: Dispersed generation impact on distribution networks. IEEE Computer Applications in Power 12(2), 22-28 (1999)

12. Dimeas, A., Hatziagyriou, N.: Operation of a multi-agent system for microgrid control. IEEE Transactions on Power Systems 20(3), 1447-1455 (2005)

13. McArthur, S., Davidson, E., Catterson, V.: Building multi-agent systems for power engineering applications. In: IEEE Power Engineering Society General Meeting 2006 (2006)

14. Rigole, T., Vanthournout, K., De Brabandere, K., Deconinck, G.: Agents controlling the electric power infrastructure. Int. Journal of Critical Infrastructures (IJCIS) 4(1/2), 96-109 (2008)

15. Dondossola, G., Lamquet, O.: Cyber risk assessment in the electric power industry. Cigré Electra Magazine 224 (2006)

16. Dondossola, G., Szanto, J., Masera, M., Fovino, I.: Effects of intentional threats to power substation control systems. Int. Journal of Critical Infrastructures (IJCIS) 4, 129-143 (2008)

17. Van Den Keybus, J., Bolsens, B., De Brabandere, K., Driesen, J.: Using a fully digital rapid prototype platform in grid-coupled power electronics applications. In: Proceedings of the 9th IEEE Conf. on Computers and Power Electronics (COMPEL 2004), Urbana-Champaign, USA (2004)

18. Xenomai: Real-Time Framework for Linux (2008), http://www.xenomai.org 